Journal of Nonlinear Mathematical Physics, Vol. 19, No. 4 (2012) 1299001 (2 pages)

DOI: $10.1142 /$ S140292511299001X

\title{
AUTHOR INDEX (Volume 19)
}

Alejo, M. A., Focusing $m K d V$

breather solutions with nonvanishing boundary condition by the inverse scattering method

Aremua, I., see Hounkonnou, M. N.

Athorne, C. \& Yilmaz, H., Laplace invariants for general hyperbolic systems

Blackmore, D. \& Prykarpatsky, A. K., The AKNS hierarchy revisited: A vertex operator approach and its lie-algebraic structure

Calogero, F., New solvable many-body model of goldfish type

Carillo, S. \& Schiebold, C., On the recursion operator for the noncommutative burgers hierarchy

Chandrasekar, V. K.,

Senthilvelan, M. \&

Lakshmanan, M., A systematic method of finding linearizing transformations for nonlinear ordinary differential equations: I. scalar case

Chandrasekar, V. K.,

Senthilvelan, M. \&

Lakshmanan, M., A

systematic method of finding

linearizing transformations for nonlinear ordinary differential equations: II. extension to coupled ODEs

Chen, S. \& Liang, K.,

Left-invariant

Pseudo-Einstein metrics on Lie groups

Chen, Y., see $\mathrm{Hu}, \mathrm{X}$.

Christov, O., On the nonlocal symmetries of the $\mu$-Camassa-Holm equation

Dai, H., see $\mathrm{Su}, \mathrm{T}$.
1 (2012) 1250009

4 (2012) 1250033

3 (2012) 1250024

1 (2012) 1250001

1 (2012) 1250006

1 (2012) 1250003

2 (2012) 1250012

2 (2012) 1250013

2 (2012) 1250015

1 (2012) 1250002

3 (2012) 1250025

4 (2012) 1250028
Demontis, F. \& van der Mee, C., Closed form solutions to the integrable discrete nonlinear Schrödinger equation

2 (2012) 1250010

Dragović, V. \& Radnović, M., Billiard algebra, integrable line congruences, and double reflection nets

Euler, M. \& Euler, N., Invariance of the Kaup-Kupershmidt equation and triangular Auto-Bäcklund transformations

Euler, N., see Euler, M.

Feng, W., Zhao, S.-L. \& Zhang, D.-J., Exact solutions to lattice Boussinesq-type equations

Frank, T. D., Nambu bracket formulation of nonlinear biochemical reactions beyond elementary mass action kinetics

Gaeta, G. \& Rodríguez, M. A., Hyperkähler structure of the taub-NUT metric

Geng, X. G., see Su, T.

Geng, X., see Gong, D.

Gong, D. \& Geng, X., Quasi-periodic solutions of the relativistic toda hierarchy

Gubbiotti, G. \& Nucci, M. C., Conservation laws for the Schrödinger-Newton equations

3 (2012) 1220001

3 (2012) 1220001

4 (2012) 1250031

1 (2012) 1250007

2 (2012) 1250014

4 (2012) 1250028

4 (2012) 1250030

4 (2012) 1250030

3 (2012) 1220002

Hounkonnou, M. N. \& Aremua, I., Landau levels in a two-dimensional noncommutative space: Matrix and quaternionic vector coherent states

4 (2012) 1250033

$\mathrm{Hu}, \mathrm{X} . \&$ Chen, Y., A direct procedure on the integrability of nonisospectral and variable-coefficient $M K d V$ equation 
Huang, L. \& Zhang, D.-J., Solutions and lax pairs based on bilinear Bäcklund transformations of some supersymmetric equations

Huang, M.-H., High-frequency asymptotics for the helmholtz equation in a half-plane

Huang, Y., see Yao, Y.

Klimčík, C., Totally classical calogero model

Kruglikov, B., Lie theorem via rank 2 distributions (integration of PDE of class $\omega=1$ )

Lakshmanan, M., see Chandrasekar, V. K.

Lakshmanan, M., see Chandrasekar, V. K.

Liang, K., see Chen, S.

Liu, Q. \& Qian, D., Construction of modulated amplitude waves via averaging in collisionally inhomogeneous Bose-Einstein condensates

Llibre, J. \& Valls, C., Darboux integrability of a simplified Friedman-Robertson-Walker hamiltonian system

Llibre, J. \& Valls, C., Liouvillian and analytic first integrals for the Brusselator system

Llibre, J. \& Valls, C., On the integrability of a Muthuswamy-Chua system

Martínez-González, A., see Rodríguez-Domínguez, A. R.

Matioc, A.-V., Steady internal water waves with a critical layer bounded by the wave surface

Nucci, M. C. \& Tamizhmani, K. M., Lagrangians for biological models

Nucci, M. C., see Gubbiotti, G.

Poletaeva, E., The first cohomology of the superconformal algebra $K(1 \mid 4)$

Prykarpatsky, A. K., see Blackmore, D.

Qian, D., see Liu, Q.

Radnović, M., see Dragović, V.
1 (2012) 1250005

1 (2012) 1250004

4 (2012) 1250027

4 (2012) 1250035

2 (2012) 1250011

2 (2012) 1250012

2 (2012) 1250013

2 (2012) 1250015

2 (2012) 1250017

4 (2012) 1250036

2 (2012) 1250016

4 (2012) 1250029

4 (2012) 1250034

1 (2012) 1250008

3 (2012) 1250021

3 (2012) 1220002

3 (2012) 1250020

1 (2012) 1250001

2 (2012) 1250017

3 (2012) 1250019
Rodríguez, M. A., see Gaeta, G.

Rodríguez-Domínguez, A. R. \& Martínez-González, A., Group of transformations with

respect to the counterpart of rapidity and related field equations

Saito, S., Octahedral structure of the Hirota-Miwa equation

Schiebold, C., see Carillo, S.

Senthilvelan, M., see Chandrasekar, V. K.

Senthilvelan, M., see Chandrasekar, V. K.

Su, T., Dai, H. \& Geng, X. G., On the application of a generalized version of the dressing method to the integration of variable coefficient $N$-coupled nonlinear Schrödinger equation

Tamizhmani, K. M., see Nucci, M. C.

Valls, C., see Llibre, J.

Valls, C., see Llibre, J.

Valls, C., see Llibre, J.

van der Mee, C., see Demontis, F.

Vekslerchik, V. E., Functional representation of the negative AKNS hierarchy

Vilasi, G., see Yanovski, A. B.

Voicu, N., Tidal tensors in the description of gravity and electromagnetism

2 (2012) 1250014

4 (2012) 1250034

4 (2012) 1250032

1 (2012) 1250003

2 (2012) 1250012

2 (2012) 1250013

4 (2012) 1250028

3 (2012) 1250021

2 (2012) 1250016

4 (2012) 1250029

4 (2012) 1250036

2 (2012) 1250010

3 (2012) 1250022

3 (2012) 1250023

2 (2012) 1250018

Watkins, J., Spectral zeta functions of a $1 D$ Schrödinger problem

Yanovski, A. B. \& Vilasi, G., Geometry of the recursion operators for the GMV system

3 (2012) 1250023

Yao, Y., Huang, Y. \& Zeng, Y., A new $\left(\gamma_{n}, \sigma_{k}\right)$-KP hierarchy and generalized dressing method

Yilmaz, H., see Athorne, C.

Zeng, Y., see Yao, Y.

Zhang, D.-J., see Feng, W.

Zhang, D.-J., see Huang, L.

Zhao, S.-L., see Feng, W.
3 (2012) 1250026

4 (2012) 1250027

3 (2012) 1250024

4 (2012) 1250027

4 (2012) 1250031

1 (2012) 1250005

4 (2012) 1250031 